\title{
INTERSECTIONS OF ANALYTICALLY AND GEOMETRICALLY FINITE SUBGROUPS OF KLEINIAN GROUPS
}

\author{
JAMES W. ANDERSON
}

\begin{abstract}
We consider the intersection of pairs of subgroups of a Kleinian group of the second kind $K$ whose limit sets intersect, where one subgroup $G$ is analytically finite and the other $J$ is geometrically finite, possibly infinite cyclic. In the case that $J$ is infinite cyclic generated by $M$, we show that either some power of $M$ lies in $G$ or there is a doubly cusped parabolic element $Q$ of $G$ with the same fixed point as $M$. In the case that $J$ is nonelementary, we show that the intersection of the limit sets of $G$ and $J$ is equal to the limit set of the intersection $G \cap J$ union with a subset of the rank 2 parabolic fixed points of $K$. Hence, in both cases, the limit set of the intersection is essentially equal to the intersection of the limit sets. The main facts used in the proof are results of Beardon and Pommerenke [4] and Canary [6], which yield that the Poincaré metric on the ordinary set of an analytically finite Kleinian group $G$ is comparable to the Euclidean distance to the limit set of $G$.
\end{abstract}

\section{INTRODUCTION}

The purpose of this paper is to investigate the intersection of certain subgroups of Kleinian groups, with an eye towards understanding the relationship between the intersection of their limit sets, which is topological information, and the limit set of their intersection, which is algebraic information. We concentrate here on Kleinian groups of the second kind, where we require one subgroup to have finite area quotient (analytic finiteness) and the other to be geometrically finite, possibly infinite cyclic. As all finitely generated Kleinian groups are analytically finite, by Ahlfors' finiteness theorem, these results apply to a large class of groups. In the case that the geometrically finite subgroup is cyclic, we obtain the following result, which is proven as Theorems 4.1 and 4.2.

Let $K$ be a Kleinian group of the second kind, let $G$ be an analytically finite subgroup of $K$, and let $M$ be an infinite order element of $K$ which has a fixed point in $\Lambda(G)$. Then, either $M^{n} \in G$ for some $n>0$ or there is a doubly cusped parabolic element $Q$ of $G$ with the same fixed point as $M$.

For a nonelementary geometrically finite subgroup, we obtain the following result, which is proven as Theorem 5.2.

Received by the editors February 26, 1992.

1991 Mathematics Subject Classification. Primary 30F40; Secondary 57M50, $20 \mathrm{H} 10$. 
Let $K$ be a Kleinian group of the second kind, let $G$ be a nonelementary analytically finite subgroup of $K$, and let $J$ be a nonelementary geometrically finite subgroup of $K$. Then, $G \cap J$ is geometrically finite and $\Lambda(G) \cap \Lambda(J)=\Lambda(G \cap J) \cup P(G, J)$. Moreover, $P(G, J) \subset \Omega(G \cap J)$.

Here, $P(G, J)$ is the set of points whose stabilizer in $K$ has rank 2 but whose stabilizers in both $G$ and $J$ have rank 1. These points are isolated in $\Omega(G \cap J)$, and are a precise measure of the difference between $\Lambda(G) \cap \Lambda(J)$ and $\Lambda(G \cap J)$.

There are two interesting features of the results proven in this paper. First, we are provided with information about how all finitely generated and some infinitely generated groups can intersect with geometrically finite groups; namely, that this intersection is always geometrically finite, and hence finitely generated. We pay a small price for this information, namely that the groups involved are subgroups of some common group of the second kind. We also get exact information about the intersection of the limit sets of the groups and its relation to the limit set of the intersection; in particular, this intersection is as large as possible, with the only exceptional points lying in the set $P(G, J)$.

Analytic finiteness is a condition which is purely planar and does not involve the 3-dimensional action of $G$. So, the proofs of these two results use information about how the groups act of the Riemann sphere $\overline{\mathbf{C}}$, making use of the Poincaré metric on the ordinary set of a nonelementary Kleinian group.

The general problem of understanding subgroups of a Kleinian group and their intersections has been investigated by a number of authors; we will attempt to give a brief chronology. Thurston showed that every finitely generated subgroup of a geometrically finite Kleinian group of the second kind is itself geometrically finite; for a proof, see [11]. Hempel [7] proved that the intersection of two finitely generated subgroups of a geometrically finite group of the second kind is again finitely generated, and hence geometrically finite. The author [2] extended this result to two finitely generated subgroups of any Kleinian group of infinite covolume. So, part of Theorem 5.2 can be thought of as extending Hempel's original result to the case of a geometrically finite and an analytically finite subgroup of a Kleinian group of the second kind.

However, we are interested in more than the group theory; we would also like to have some concrete information about the relationship between the action of the groups on the Riemann sphere and their group theory. This line of work also has a history. Maskit [9] proved the analogues of the two main theorems given here in the case that the subgroups are component subgroups of a Kleinian group; in this case, the set $P(G, J)$ is empty.

If we remove the assumption that the groups be of the second kind, stronger hypotheses are needed on the subgroups involved. Susskind [13] proved the analogues of the two main theorems in the case that the subgroups are geometrically finite; these results were extended to higher dimensions by Susskind and Swarup [14]. Soma [12] showed that the conclusions of both the main theorems hold for two function groups which are subgroups of a Kleinian group. These results were extended by the author [1] to any pair of topologically tame subgroups of a Kleinian group. 


\section{ACKNOWLEDGMENTS}

The first result is the main result of my doctoral thesis from SUNY Stony Brook; I would like to thank my advisor, Bernie Maskit, for his support and encouragement. The second result is work done while I was a postdoctoral fellow at the Mathematical Sciences Research Institute in Berkeley; this work was supported in part by NSF grant DMS 8505550. I would also like to thank Dick Canary for several helpful discussions and the referee for several careful readings of the early drafts and for pointing out that the original proof of Corollary 3.4 was incorrect.

\section{Preliminaries}

In this section, we define our terms and present some basic results we will make use of later. Our reference for the basics of Kleinian groups is [10].

A Kleinian group $G$ is a discrete subgroup of $P S L_{2}(\mathbf{C})$, which we consider as acting on the Riemann sphere $\overline{\mathbf{C}}$ by Möbius transformations. The action of $G$ decomposes $\overline{\mathbf{C}}$ into two disjoint sets. The ordinary set $\Omega(G)$ is the set of points of $\overline{\mathbf{C}}$ at which $G$ acts discontinuously; if $\Omega(G)$ is nonempty, we say that $G$ is of the second kind. The connected components of $\Omega(G)$ are the components of $G$. Let $\Omega^{0}(G)$ denote $\Omega(G)$ minus the fixed points of elements of finite order.

The complement of $\Omega(G)$ in $\overline{\mathbf{C}}$ is the limit set, denoted $\Lambda(G)$. A Kleinian group $G$ is nonelementary if $\Lambda(G)$ contains at least 3 points; otherwise, $G$ is elementary. An elementary group is either finite with empty limit set, contains a purely parabolic subgroup of finite index and has limit set a single point, or contains a loxodromic cyclic subgroup of finite index and has limit set a pair of points. For a complete description of elementary groups, see [10]. Alternatively, for a nonelementary $G$, we can think of $\Lambda(G)$ as the set of accumulation points of the orbit of any point in $\overline{\mathbf{C}}$ under $G$.

For any set $X$ in $\overline{\mathbf{C}}$, define the stabilizer of $X$ in $G$ to be the subgroup $\operatorname{stab}_{G}(X)=\{g \in G: g(X)=X\}$ of elements of $G$ which keep $X$ invariant; the stabilizer of a component $\Delta$ of $G$ is denoted $G_{\Delta}$. For a subgroup $H$ of $G$, we say $X$ is precisely invariant under $H$ in $G$ if $h(X)=X$ for all $h \in H$ and $g(X) \cap X$ is empty for all $g \in G-H$.

Let $T$ be a Möbius transformation which does not fix $\infty$. The isometric circle $I_{T}$ of $T$ is the circle $\left\{z \in \mathrm{C}:\left|T^{\prime}(z)\right|=1\right\}$; the radius of $I_{T}$ is $r(T)$ and the center of $I_{T}$ is $T^{-1}(\infty)$. The following lemma of Shimizu and Leutbecher lays out constraints on the radii of isometric circles of elements in certain Kleinian groups.

Lemma 2.1 [10]. Let $G$ be a Kleinian group containing $P(z)=z+1$ and let $g$ be an element of $G$ not fixing $\infty$. Then, $r(g) \leq 1$.

For a Kleinian group $G$, let $\operatorname{ext}(G)$ denote the common exteriors of the isometric circles of all elements of $G$ not fixing $\infty$. If $g$ is an element of $G$ not fixing $\infty$, then $g(\operatorname{ext}(G))$ is disjoint from $\operatorname{ext}(G)$, as $g$ maps $I_{g}$ to $I_{g^{-1}}$, taking the region exterior to $I_{g}$ onto the region interior to $I_{g^{-1}}$.

A point of approximation of $G$ is a limit point of $G$ which behaves like a fixed point of a loxodromic element of $G$. More precisely, we say $x$ is a point 
of approximation of $G$ if there exist infinitely many distinct elements $g_{m}$ of $G$ so that, for every compact set $C$ in $\overline{\mathbf{C}}-\{x\}$, there is a constant $\epsilon_{C}>0$ so that $\left|g_{m}(x)-g_{m}(z)\right| \geq \epsilon_{C}>0$ for all $z \in C$ and all the $g_{m}$. It is easy to see that a fixed point of a loxodromic element of $G$ is a point of approximation. We will also need to make use of an alternative definition of point of approximation.

Lemma 2.2 [10]. Let $G$ be a Kleinian group with $\infty \in \Omega^{0}(G)$, and let $x$ be a point of $\Lambda(G)$. Suppose there exist infinitely many distinct elements $g_{m}$ of $G$ so that $\left|g_{m}(x)-g_{m}(z)\right| \geq \epsilon_{C}>0$ on compact subsets $C$ of $\overline{\mathbf{C}}-\{x\}$. Then, there is a constant $A$ so that $\left|x-g_{m}^{-1}(\infty)\right| \leq A r\left(g_{m}\right)^{2}$.

We say that a parabolic element $P$ of $G$ fixing a point $x$ is doubly cusped if there exist two open circular discs $D_{1}$ and $D_{2}$ in $\Omega(G)$ so that $D_{1} \cup D_{2}$ is precisely invariant under $\operatorname{stab}_{G}(x)$ in $G$. The discs $D_{j}$ are the cusp regions of $P$.

For subgroups $J$ and $H$ of $G$, define $P(J, H)$ to be those points $x$ of $\Lambda(G)$ so that $\left\langle\operatorname{stab}_{J}(x), \operatorname{stab}_{H}(x)\right\rangle$ has rank 2 and both $\operatorname{stab}_{J}(x)$ and $\operatorname{stab}_{H}(x)$ have rank 1.

There are two basic results which describe how infinite order elements of Kleinian groups can share fixed points; the proofs of both are calculations which can be found in [10].

Proposition 2.3 [10]. Let $G$ be a Kleinian group. Then, no point of $\overline{\mathbf{C}}$ can be fixed by both a parabolic element of $G$ and a loxodromic element of $G$.

Proposition 2.4 [10]. Let $G$ be a Kleinian group and let $L$ and $M$ be loxodromic elements of $G$. If $L$ and $M$ share a fixed point, then $L^{n}=M^{k}$ for some pair $n$ and $k$ of nonzero integers.

A Kleinian group is said to be geometrically finite if there exists a finite sided fundamental polyhedron for its action on hyperbolic 3-space $\mathbf{H}^{3}$. The following theorem of Beardon and Maskit restates this property in terms of the limit set.

Theorem 2.5 [3]. A Kleinian group $G$ is geometrically finite if and only if every point of $\Lambda(G)$ is either a rank 2 parabolic fixed point, a doubly cusped parabolic fuxed point, or a point of approximation.

\section{The Poincaré Metric}

The Poincaré metric is a canonical complete metric of constant curvature -1 which is defined on any domain $\Omega$ in $\overline{\mathbf{C}}$ whose boundary $\partial \Omega$ contains at least 3 points; we say such a domain is hyperbolic. On that part of $\Omega$ which lies in the complex plane, we can express the line element of the Poincare metric as $\rho_{\Omega}(z)|d z|$, where $z$ is the standard complex coordinate on the plane. Let $\delta_{\Omega}(z)$ denote the Euclidean distance from $z$ to $\partial \Omega$. The most general result concerning the interaction of $\delta_{\Omega}$ and $\rho_{\Omega}$ is due to Beardon and Pommerenke.

Theorem 3.1 [4]. For a hyperbolic subdomain $\Omega$ of $\mathrm{C}$, we have that

$$
\frac{1}{2 \sqrt{2}} \leq \delta_{\Omega}(z) \rho_{\Omega}(z)\left(k+\beta_{\Omega}(z)\right) \leq k+\frac{\pi}{4},
$$


where $k=4+\log (3+2 \sqrt{2})$ and

$$
\beta_{\Omega}(z)=\inf \left\{|\log | \frac{z-a}{b-a}||: a \in \partial \Omega, b \in \partial \Omega,|z-a|=\delta_{\Omega}(z)\right\} .
$$

Hence, if it is possible to bound $\beta_{\Omega}(z)$ from above on a subset $S$ of a hyperbolic subdomain $\Omega$ of $\mathbf{C}$, then there is a lower bound on the product $\delta_{\Omega}(z) \rho_{\Omega}(z)$ for all $z \in S$. In our situation, we will make use of the following lemma of Canary, which provides an explicit connection between the geometry of $\Omega$ and $\beta_{\Omega}$.

Lemma 3.2 [6]. Let $\Omega$ be a hyperbolic subdomain of $\mathbf{C}$. If

$$
\beta_{\Omega}\left(z^{\prime}\right) \geq M>\log (2),
$$

then there is a homotopically nontrivial curve $g$ through $z^{\prime}$ whose length in the Poincaré metric on $\Omega$ is at most $\left(8 \pi k+2 \pi^{2}\right) / M$.

Consider now a nonelementary Kleinian group $G$; each component of $G$ is then a hyperbolic domain, and the elements of $G$ act on $\Omega(G)$ as isometries in the Poincaré metric. We will let $\delta_{G}=\delta_{\Omega(G)}$ and $\rho_{G}=\rho_{\Omega(G)}$. We say that $G$ is analytically finite if the area of the quotient $\Omega(G) / G$ is finite. The area of $\Omega(G) / G$ is the same as the area of a fundamental domain for the action of $G$ on $\Omega(G)$; hence, an equivalent formulation of analytic finiteness is that any open set in $\Omega(G)$ which is precisely invariant under the identity in $G$ has finite area.

Ahlfors' finiteness theorem [8] states that every finitely generated Kleinian group is analytically finite; there are explicit examples (see [10]) to show that the converse is false. By convention, elementary groups are also considered to be analytically finite.

Let $G$ be a nonelementary analytically finite Kleinian group and let $c_{0}$ be the length of the shortest closed geodesic on $\Omega(G) / G$. Let $\Delta$ be a component of $G$ not containing $\infty$. Since every homotopically nontrivial loop $\gamma$ on $\Delta$ descends to a homotopically nontrivial loop on $\Delta / G_{\Delta}$, the length of $\gamma$ is bounded below by $c_{0}$. Hence, if no component of $G$ contains $\infty$, we obtain the following.

Corollary 3.3 [6]. Let $G$ be a nonelementary analytically finite Kleinian group of the second kind with $\infty \in \Lambda(G)$. There exists a positive constant $a$, depending on $G$, so that $\delta_{G}(z) \rho_{G}(z) \geq a$ for all $z \in \Omega(G)$.

We will also need to examine the case that there is a component $\Delta^{\prime}$ containing $\infty$. In this case, we obtain the following result.

Corollary 3.4. Let $G$ be a nonelementary analytically finite Kleinian group of the second kind with $\infty \in \Omega^{0}(G)$. For each choice of $R>0$ so that $D=\{|z| \geq R\}$ lies in $\Omega(G)$, there exists a positive constant $a$, depending on $G$ and $R$, so that $\delta_{G}(z) \rho_{G}(z) \geq a$ for all $z \in \Omega(G)-D$.

Proof. Let $A$ be the Euclidean diameter of $\Lambda(G)$. Given a point $z \in \Omega(G)-$ $D$, let $z_{1}$ be a point of $\Lambda(G)$ which is closest to $z$; in particular, we have that $\left|z-z_{1}\right|=\delta_{G}(z)$. 
Now, choose a point $z_{0} \in \Lambda(G)$ so that both $\left|z-z_{0}\right| \geq \frac{1}{6} A$ and $\left|z_{1}-z_{0}\right| \geq$ $\frac{1}{6} A$. Such a point $z_{0}$ always exists: If $\delta_{G}(z)>\frac{1}{6} A$, then $\left|z-z_{0}\right|>\frac{1}{6} A$ for all $z_{0} \in \Lambda(G)$, so choose any $z_{0}$ with $\left|z_{0}-z_{1}\right| \geq \frac{1}{6} A$. If $\delta_{G}(z) \leq \frac{1}{6} A$, choose any $z_{0}$ so that $\left|z_{1}-z_{0}\right| \geq \frac{1}{3} A$; then, we have that $\left|z-z_{0}\right| \geq \frac{1}{6} A$ by the triangle inequality.

Let $P$ be the parabolic Möbius transformation which fixes $z_{0}+2 R$ and which takes $z_{0}$ to $\infty$ :

$$
P(z)=\frac{\left[1+\frac{1}{2 R}\left(z_{0}+2 R\right)\right] z-\frac{1}{2 R}\left(z_{0}+2 R\right)^{2}}{\frac{1}{2 R} z-\frac{1}{2 R} z_{0}} .
$$

An easy calculation shows that

$$
P^{\prime}(z)=\frac{4 R^{2}}{\left(z-z_{0}\right)^{2}}
$$

in particular, we have that $1 \leq\left|P^{\prime}(z)\right|$, since neither $z$ nor $z_{0}$ lies in $D$.

Let $G^{\prime}=P G P^{-1}$; then, we have that $\Omega\left(G^{\prime}\right)=P(\Omega(G))$ and $\Lambda\left(G^{\prime}\right)=$ $P(\Lambda(G))$. Let $\rho_{G^{\prime}}(z)|d z|$ be the line element of the Poincaré metric on $\Omega\left(G^{\prime}\right)$, where $z$ is the standard coordinate on $C$, and let $\delta_{G^{\prime}}(z)$ be the Euclidean distance to $\Lambda\left(G^{\prime}\right)$.

Since $\infty \in \Lambda\left(G^{\prime}\right)$ and $G^{\prime}$ is analytically finite, there exists a positive constant $a^{\prime}$ so that $\delta_{G^{\prime}}(z) \rho_{G^{\prime}}(z) \geq a^{\prime}$ for all $z \in \Omega\left(G^{\prime}\right)$, by Corollary 3.3. This constant $a^{\prime}$ depends only on the length of the shortest homotopically nontrivial curve on $\Omega(G) / G$, and so is independent of the choice of $z_{0}$ and $P$.

$P$ is a conformal homeomorphism taking $\Omega(G)$ to $\Omega\left(G^{\prime}\right)$, and so preserves the Poincaré metric; hence, setting $w=P(z)$, we have that $\rho_{G}(z)|d z|$ $=\rho_{G^{\prime}}(w)|d w|$. Since $\rho_{G^{\prime}}(w)|d w|=\rho_{G^{\prime}}(P(z))\left|P^{\prime}(z)\right||d z|$, this yields

$$
\rho_{G^{\prime}}(P(z))=\frac{1}{\left|P^{\prime}(z)\right|} \rho_{G}(z) \leq \rho_{G}(z) .
$$

Both $\left|P^{\prime}(z)\right|$ and $\left|P^{\prime}\left(z_{1}\right)\right|$ are bounded above by $(12 R / A)^{2}$, by our choice of $z_{0}$. Hence, an upper bound on $\delta_{G^{\prime}}(P(z))$ is given by

$$
\delta_{G^{\prime}}(P(z)) \leq\left|P(z)-P\left(z_{1}\right)\right|=\left|P^{\prime}(z) P^{\prime}\left(z_{1}\right)\right|^{1 / 2}\left|z-z_{1}\right| \leq\left(\frac{12 R}{A}\right)^{2} \delta_{G}(z) .
$$

Hence,

$$
a^{\prime} \leq \delta_{G^{\prime}}(P(z)) \rho_{G^{\prime}}(P(z)) \leq\left(\frac{12 R}{A}\right)^{2} \rho_{G}(z) \delta_{G}(z)
$$

Taking $a=(A / 12 R)^{2} a^{\prime}$, we are done.

These two corollaries are saying essentially the same thing, though we are normalizing the groups differently. We will most often use these corollaries in conjunction with the following lemma.

Lemma 3.5. Let $K$ be a Kleinian group of the second kind and let $G$ be a nonelementary analytically finite subgroup of $K$. Suppose there exists an open set $D$ in $\Omega(K)$ precisely invariant under the identity in $K$ and a sequence $\left\{k_{m}\right\}$ of distinct elements of $K$ so that the areas of the $k_{m}(D)$ in the Poincare metric 
on $\Omega(G)$ are uniformly bounded away from zero. Then, for some fixed $m$, we have that $k_{m} \cdot k_{n}^{-1} \in G$ for infinitely many $n$.

Proof. Project the $k_{m}(D)$ to $\Omega(G) / G$. The precise invariance of $D$ under the identity in $K$ implies that the projections of the $k_{n}(D)$ coincide if they overlap. Since $\Omega(G) / G$ has finite area, the projections of infinitely many of the $k_{n}(D)$ must then coincide and so $k_{n} \cdot k_{m}^{-1} \in G$ for some fixed $m$ and infinitely many $n$.

Of particular interest is the case that $k_{m}=M^{m}$ for some $M \in K$; here, we get that $M^{k} \in G$ for some $k>0$. It is worth noting that Corollaries 3.3 and 3.4 imply that the Poincare areas of the $k_{m}(D)$ will be uniformly bounded away from zero if they lie a uniformly bounded distance from $\Lambda(G)$ and have Euclidean areas uniformly bounded from below.

We will make use of the Poincare metric in a second way, namely the following.

Lemma 3.6. Let $G$ be a nonelementary analytically finite Kleinian group of the second kind with $\infty \in \Lambda(G)$. If $\operatorname{ext}(G)$ has infinite area in the Poincaré metric on $\Omega(G)$, there is an infinite order element $Q$ of $G$ fixing $\infty$.

Proof. Let $H$ denote the stabilizer of $\infty$ in $G$. If $H$ is finite, it is cyclic of order $k$, and the projection of $\operatorname{ext}(G)$ to $\Omega(G) / G$ is at most $k$-to-one. This implies that the projection of $\operatorname{ext}(G)$ to $\Omega(G) / G$ has infinite Poincare area, which contradicts the analytic finiteness of $G$.

\section{ELEMENTARY INTERSECTIONS}

In this section, we consider the intersection of an analytically finite subgroup and an infinite cyclic subgroup of a Kleinian group of the second kind, where the limit sets of the subgroups intersect. We consider first the case that the cyclic subgroup is loxodromic cyclic.

Theorem 4.1. Let $K$ be a Kleinian group of the second kind, let $G$ be an analytically finite subgroup of $K$, and let $L$ be a loxodromic element of $K$ which has a fixed point in $\Lambda(G)$. Then, $L^{n} \in G$ for some $n>0$.

Proof. We begin with the case that $G$ is elementary. We have assumed that $\Lambda(G)$ is nonempty, so $G$ cannot be finite. By Proposition 2.3, $G$ cannot contain a purely parabolic subgroup of finite index. If $G$ contains a loxodromic cyclic subgroup of finite index, Proposition 2.4 implies that $L^{n} \in G$ for some $n>0$.

Assume now that $G$ is nonelementary; all Poincare areas are measured using the Poincare metric on $\Omega(G)$. Let $x_{0}$ be a fixed point of $L$ contained in $\Lambda(G)$. Without loss of generality, we can assume that $L(z)=\lambda z, x_{0}=\infty$, and $1 \in \Lambda(G)$. Since $1 \in \Lambda(G)$, we have that $\delta_{G}(z) \leq|z-1|$. If necessary, replace $L$ by $L^{-1}$ so that $|\lambda|>1$.

Let $D$ be an open disc in $\Omega(K)$ which is precisely invariant under the identity in $K$. For $m$ sufficiently large, $L^{m}(D)$ lies outside the unit circle and so $\delta_{G}(z)<2|z|$ on $L^{m}(D)$. 
Using this and Corollary 3.3 , we have that

$$
\int_{L^{m}(D)} \rho_{G}^{2}(z) d A \geq \frac{a^{2}}{4} \int_{L^{m}(D)} \frac{1}{|z|^{2}} d A .
$$

A standard change of variables calculation shows that

$$
\int_{L^{m}(D)} \frac{1}{|z|^{2}} d A=\int_{D} \frac{1}{|z|^{2}} d A
$$

Hence, for $m$ sufficiently large, the $L^{m}(D)$ have Poincare areas uniformly bounded away from zero, and Lemma 3.5 implies then that $L^{n} \in G$ for some $n>0$.

In the case that the cyclic subgroup is parabolic cyclic, there are two possible outcomes, both of which can occur. The proof here is complicated by the fact that we cannot use the same argument as in the loxodromic case, as the Euclidean areas of the translates of a set by powers of a parabolic fixing $\infty$ are constant.

Theorem 4.2. Let $K$ be a Kleinian group of the second kind, let $G$ be an analytically finite subgroup of $K$, and let $P$ be a parabolic element of $K$ whose fixed point lies in $\Lambda(G)$. Either $P^{n} \in G$ for some $n>0$ or there exists a doubly cusped parabolic element $Q$ of $G$ with the same fixed point as $P$.

Proof. First, we consider the case that $G$ is elementary. Since $\Lambda(G)$ is assumed to be nonempty, $G$ cannot be finite. By Proposition 2.3, $G$ cannot contain a loxodromic cyclic subgroup of finite index.

The remaining case is that $G$ contains a purely parabolic subgroup $H$ of finite index. Either $P^{n} \in G$ for some $n>0$ or $H$ has rank 1, as Kleinian groups cannot contain $\mathbf{Z} \oplus \mathbf{Z} \oplus \mathbf{Z}$ subgroups. In the latter case, every element of $H$ is doubly cusped.

Assume now that $G$ is nonelementary; all Poincare areas will be measured using the Poincare metric on $\Omega(G)$. Normalize $K$ so that $P(z)=z+1$; Lemma 2.1 then implies that $\operatorname{ext}(G)$ contains the set $E=\left\{\delta_{G}(z)>1\right\}$, as the center $g^{-1}(\infty)$ of each isometric circle lies in $\Lambda(G)$.

We begin by showing that there exists a parabolic element $Q$ of $G$ fixing $\infty$. For each $n \geq 0$, let $V_{n}$ be the closed vertical strip $\{n \leq \operatorname{Re}(z) \leq n+1\}$. Choose an open disc $D$ contained in $V_{0}$ which is precisely invariant under the identity in $K$, and note that the Euclidean areas of the $P^{n}(D)$ are constant.

If infinitely many of the $P^{n}(D)$ lie a uniformly bounded Euclidean distance from $\Lambda(G)$, then Lemma 3.5 immediately implies that $P^{k} \in G$ for some $k>0$.

Suppose that only finitely many of the $V_{n}$ contain points of $\Lambda(G)$; then, some half plane $\{\operatorname{Re}(z)>M\}$ lies in $E$, as the points of $\Lambda(G)$ all lie in some finite width vertical strip. An easy calculation using Corollary 3.3 shows that this half plane has infinite Poincare area. By Lemma 3.6, there exists an infinite order element $Q$ of $G$ fixing $\infty$; using Proposition 2.3, we see that $Q$ must be parabolic.

The remaining case is that infinitely many of the $V_{n}$ contain points of $\Lambda(G)$ and the Euclidean distances from the $P^{n}(D)$ to $\Lambda(G)$ are not bounded on any subsequence of the $n$. Hence, we can choose a subsequence $V_{n_{j}}$ of the $V_{n}$ so that the Euclidean distances from the $P^{n_{j}}(D)$ to $\Lambda(G)$ are increasing 
monotonically as $n_{j}$ goes to infinity. For each $n_{j}$, choose a point $z_{n_{j}}$ in $V_{n_{j}}$ so that $\delta\left(z_{n_{j}}\right)=1$ and $\left|\operatorname{Im}\left(z_{n_{j}}\right)\right| \leq|\operatorname{Im}(z)|$ for all points $z$ in $V_{n_{j}}$ with $\delta_{G}(z)=1$; the $z_{n_{j}}$ are points in $V_{n_{j}}$ which have minimal imaginary part among all points $z$ in $V_{n_{j}}$ with $\delta_{G}(z)=1$. Such $z_{n_{j}}$ exist for $n_{j}$ sufficiently large, as the Euclidean distances from the $P^{n_{j}}(D)$ to $\Lambda(G)$ are increasing. Moreover, the $\left|\operatorname{Im}\left(z_{n_{j}}\right)\right|$ are not bounded on any subsequence; in particular, we have that $\left|\operatorname{Im}\left(z_{n_{j}}\right)\right| \geq 1$ for $n_{j}$ sufficiently large. By our choice of $z_{n_{j}}$, the set

lies in $E$.

$$
A_{n_{j}}=\left\{z \in V_{n_{j}}:|\operatorname{Im}(z)|<\left|\operatorname{Im}\left(z_{n_{j}}\right)\right|\right\}
$$

Calculating using Corollary 3.3, we see that the Poincare areas of the $A_{n_{j}}$ are bounded away from zero for $n_{j}$ sufficiently large, as each such $A_{n_{j}}$ contains a Euclidean square of side length 1 with $z_{n_{j}}$ on its boundary. So, $\operatorname{ext}(G)$ has infinite Poincare area and Lemma 3.2 then implies the existence of a parabolic element $Q$ of $G$ fixing $\infty$.

Let $H$ be the stabilizer of $\infty$ in $G$; if $H$ has rank 2, then the discreteness of $K$ forces $P^{k} \in G$ for some $k>0$. Hence, we can assume that $H$ has rank 1; without loss of generality, we assume $Q$ is primitive and write $Q(z)=z+\tau$. If $\tau$ is real, then the discreteness of $K$ implies that $P^{k} \in G$ for some $k>0$.

Otherwise, let $l_{n}$ be the line through $n$ and $n+\tau$ and let $V_{n}$ be the strip between $l_{n}$ and $l_{n+1}$. If $Q$ is not doubly cusped, then infinitely many $V_{n_{j}}$ of the $V_{n}$ contain a point $w_{n_{j}}$ of $\Lambda(G)$. Translating $w_{n_{j}}$ by an appropriate power of $Q$, we can assume that $\left|\operatorname{Im}\left(w_{n_{j}}\right)\right| \leq|\operatorname{Im}(\tau)|$ for all $n_{j}$. Hence, the Euclidean distances of the $w_{n_{j}}$ to $P^{n_{j}}(D)$ are uniformly bounded from above and so Lemma 3.5 implies that $P^{k} \in G$ for some $k>0$.

As a corollary to these two theorems, we have the following.

Corollary 4.3. Let $K$ be a Kleinian group of the second kind and let $G$ be a nonelementary analytically finite subgroup. Suppose that there is an element $k \in K$ of infinite order with $k(\Lambda(G)) \subset \Lambda(G)$. Then, $k^{n} \in G$ for some $n>0$ and $k(\Lambda(G))=\Lambda(G)$.

Proof. Since $k(\Lambda(G)) \subset \Lambda(G)$, we have that $k^{n}(\Lambda(G)) \subset \Lambda(G)$ for all $n>$ 0 . If $k(\Lambda(G)) \neq \Lambda(G)$, then $k^{n}(\Lambda(G)) \neq \Lambda(G)$ for all $n>0$. Hence, if $k^{n} \in G$ for some $n>0$, then $k^{n}(\Lambda(G))=\Lambda(G)$ and so $k(\Lambda(G))=\Lambda(G)$.

Note that the fixed point set of $k$ lies in $\Lambda(K)$; if not, then some sufficiently high power $k^{m}$ of $k$ would satisfy $k^{m}(\Lambda(G)) \cap \Lambda(G)=\emptyset$. If $k$ is loxodromic, then Theorem 4.1 immediately implies that some power of $k$ belongs to $G$.

If $k$ is parabolic, then, by Theorem 4.2 , either $k^{n} \in G$ for some $n>0$ or there is a doubly cusped parabolic element $Q \in G$ with the same fixed point as $k$. The latter case cannot occur; this is easist to see if we normalize so that $Q(z)=z+1$. Then, $k(z)=z+\tau$, where $\operatorname{Im}(\tau) \neq 0$, and $\Lambda(G) \subset\{|\operatorname{Im}(z)|<$ c\} for some constant $c>0$. Hence, $k^{m}(\Lambda(G)) \cap \Lambda(G)=\{\infty\}$ for all $m$ sufficiently large, a contradiction.

\section{NONELEMENTARY INTERSECTIONS}

In this section, we consider the intersection of a nonelementary analytically finite subgroup and a nonelementary geometrically finite subgroup of a Kleinian 
group of the second kind. We begin with the following proposition, which is of independent interest. As in the previous section, the proof involves estimating the Poincaré areas in $\Omega(G)$ of translates of a disc by elements of $J$.

Proposition 5.1. Let $K$ be a Kleinian group of the second kind, let $G$ be a nonelementary analytically finite subgroup of $K$, let $J$ be any nonelementary subgroup of $K$, and let $x$ be a point of $\Lambda(G) \cap \Lambda(J)$ which is a point of approximation of $J$. Then, $x \in \Lambda(G \cap J)$ and $x$ is a point of approximation of $G \cap J$.

Proof. Normalize $K$ so that $\infty \in \Omega^{0}(G)$ and $x=0$; choose $R$ so that the disc $D=\{|z|>R\}$ is precisely invariant under the identity in $K$ and the closure of $D$ lies in $\Omega(K)$. All Poincaré areas are calculated using the Poincaré metric on $\Omega(G)$.

Since 0 is a point of approximation of $J$, there are infinitely many distinct elements $j_{m}$ in $J$ so that $\left|j_{m}(0)-j_{m}(z)\right| \geq \epsilon_{C}>0$ on compact subsets $C$ of $\overline{\mathbf{C}}-\{0\}$; by Lemma 2.2 , there is then a constant $A>0$ so that $\left|j_{m}^{-1}(\infty)\right| \leq$ $\operatorname{Ar}\left(j_{m}\right)^{2}$ for all $m$.

Let $e_{m}$ be the Euclidean distance from $j_{m}(\infty)$ to $D$. Any accumulation point of the $j_{m}(\infty)$ is a point of $\Lambda(K)$ and the closure of $D$ is contained in $\Omega(K)$, so the $e_{m}$ are uniformly bounded away from zero. That is, there is a positive constant $e$ so that $e_{m} \geq e$ for all $m$.

Using an estimate on page 11 of [10], we then have that

$$
\frac{1}{R} r\left(j_{m}\right)^{2} \leq \operatorname{diam}_{E}\left(j_{m}^{-1}(D)\right) \leq \frac{2}{e} r\left(j_{m}\right)^{2}
$$

for all $m$, where $\operatorname{diam}_{E}(X)$ denotes the Euclidean diameter of $X$. In particular, we have that

$$
\int_{j_{m}^{-1}(D)} d A \geq \frac{\pi}{4 R^{2}} r\left(j_{m}\right)^{4}
$$

Since all the $j_{m}^{-1}(D)$ lie in $\{|z|<R\}$, this implies that the $r\left(j_{m}\right)$ must go to zero; consequently, the $j_{m}^{-1}(\infty)$ converge to 0 .

For $z \in j_{m}^{-1}(D)$, we have that

$$
\delta_{G}(z) \leq\left|j_{m}^{-1}(\infty)\right|+\operatorname{diam}_{E}\left(j_{m}^{-1}(D)\right) \leq B r\left(j_{m}\right)^{2},
$$

where $B=A+2 / e$ is constant. Using this and Corollary 3.4 , we have that

$$
\int_{j_{m}^{-1}(D)} \rho_{G}^{2}(z) d A \geq \frac{a^{2}}{B^{2} r\left(j_{m}\right)^{4}} \int_{j_{m}^{-1}(D)} d A \geq \frac{a^{2} \pi}{4 B^{2} R^{2}}
$$

and so the Poincare areas of the $j_{m}^{-1}(D)$ are uniformly bounded away from zero.

By Lemma 3.5, we have that $g_{m}=j_{m}^{-1} \cdot j_{m_{0}}$ is an element of $G$ for infinitely many $m$ and some fixed $m_{0}$. Therefore, $g_{m}$ is an element of $G \cap J$; since the $g_{m}\left(j_{m_{0}}^{-1}(\infty)\right)$ converge to 0 , we have that $0 \in \Lambda(G \cap J)$.

We need now to show that 0 is a point of approximation of $G \cap J$. Recall that $\left|j_{m}(0)-j_{m}(z)\right| \geq \epsilon_{C}>0$ on compact subsets $C$ of $\overline{\mathbf{C}}-\{0\}$. The map $j_{m_{0}}^{-1}$ is a homeomorphism of $\overline{\mathbf{C}}$ and so, for each compact subset $C$ of $\overline{\mathbf{C}}-\{0\}$, we can find a constant $\eta_{C}>0$ so that $\left|j_{m_{0}}^{-1} \cdot j_{m}(0)-j_{m_{0}}^{-1} \cdot j_{m}(z)\right| \geq \eta_{C}>0$ on $C$. Since the $g_{m}^{-1}=j_{m_{0}}^{-1} \cdot j_{m}$ are elements of $G \cap J$, we are done. 
We are now ready for the case of a general geometrically finite subgroup.

Theorem 5.2. Let $K$ be a Kleinian group of the second kind, let $G$ be a nonelementary analytically finite subgroup of $K$, and let $J$ be a nonelementary geometrically finite subgroup of $K$. Then, $G \cap J$ is geometrically finite and $\Lambda(G) \cap \Lambda(J)=\Lambda(G \cap J) \cup P(G, J)$. Moreover, $P(G, J) \subset \Omega(G \cap J)$.

Proof. It is easy to see that $\Lambda(G \cap J) \cup P(G, J) \subset \Lambda(G) \cap \Lambda(J)$. Now, let $x \in \Lambda(G) \cap \Lambda(J)$ be any point. Since $J$ is geometrically finite, Theorem 2.5 gives that $x$ is either a point of approximation of $J$, a rank 2 parabolic fixed point of $J$, or is a doubly cusped parabolic fixed point of $J$.

If $x$ is a point of approximation of $J$, Proposition 5.1 gives that $x$ is a point of approximation of $G \cap J$. If $x$ is a parabolic fixed point of $J$, the stabilizer of $x$ in $J$ is either rank 2 or is rank 1 and doubly cusped. In this case, we can use Theorem 4.2 to see that the stabilizer of $x$ in $G$ is also either rank 2 or is rank 1 and doubly cusped; we see this by adjoining to $G$ the parabolic elements of $J$ fixing $x$.

If $x$ is a rank 2 parabolic fixed point of both $G$ and $J$, then the discreteness of $K$ forces $x$ to be a rank 2 parabolic fixed point of $G \cap J$. If $x$ is a rank 2 fixed point of $G$ and is a doubly cusped fixed point of $J$ (or vice versa), then $x$ is a doubly cusped fixed point of $G \cap J$. If $x$ is a doubly cusped fixed point of both $G$ and $J$ and the stabilizers of $x$ in $G$ and $J$ together generate a rank 1 subgroup of $K$, then $x$ is a doubly cusped fixed point of $G \cap J$.

However, it may be that $x$ is a doubly cusped fixed point of both $G$ and $J$ and the stabilizers of $x$ in $G$ and $J$ together generate a rank 2 subgroup of $K$. These are precisely the points that make up $P(G, J)$.

To summarize, we have shown that every point of $\Lambda(G) \cap \Lambda(J)$ is a point of either $\Lambda(G \cap J)$ or $P(G, J)$. Further, we have shown that every point of $\Lambda(G \cap J)$ is either a point of approximation, a rank 2 parabolic fixed point, or a doubly cusped rank 1 parabolic fixed point. Invoking Theorem 2.5 , we see that $G \cap J$ is geometrically finite.

All that remains to show is that $P(G, J) \subset \Omega(G \cap J)$; this argument is contained in [13], but we present it here for the sake of completeness. Let $x$ be any point of $P(G, J)$, and recall that $x$ is a doubly cusped parabolic fixed point of both $G$ and $J$. The union of the cusp regions for $G$ and $J$ at $x$ fills out a neighborhood of $x$; this is easiest to see if we normalize so that $x=\infty$. So, points of $\Lambda(G \cap J)$ cannot accumulate to $x$. Since $\Omega(G) \cup \Omega(J) \subset \Omega(G \cap J)$ and limit sets are perfect (that is, every point is an accumulation point), it must be that $x$ lies in $\Omega(G \cap J)$.

\section{REFERENCES}

1. J. W. Anderson, Intersections of topologically tame subgroups of Kleinian groups, preprint.

2. $\_$, On the finitely generated intersection property for Kleinian groups, Complex Variables 17 (1991), 111-112.

3. A. Beardon and B. Maskit, Limit points of Kleinian groups and finite sided fundamental polyhedra, Acta Math. 132 (1974), 1-12.

4. A. Beardon and C. Pommerenke, The Poincaré metric of plane domains, J. London Math. Soc. (2) 18 (1978), 475-483.

5. R. Canary, Covering theorems for hyperbolic 3-manifolds, preprint. 
6. The Poincaré metric and a conformal version of a theorem of Thurston, Duke Math. J. 64 (1991), 349-359.

7. J. Hempel, The finitely generated intersection property for Kleinian groups, Knot Theory and Manifolds, (D. Rolfsen, ed.), Lecture Notes in Math., vol. 1144, Springer-Verlag, Berlin and New York, 1985.

8. I. Kra, Automorphic forms and Kleinian groups, Benjamin, Reading, MA, 1972.

9. B. Maskit, Intersections of component subgroups of Kleinian groups, Discontinuous Groups and Riemann Surfaces, (L. Greenberg, ed.), Ann. of Math. Stud., no. 79, Princeton Univ. Press, Princeton, NJ, 1974.

10. __ Kleinian groups, Springer-Verlag, Berlin, 1988.

11. J. Morgan, On Thurston's uniformization theorem for three-dimensional manifolds, The Smith Conjecture (H. Bass and J. Morgan, eds.), Academic Press, San Diego, 1984.

12. T. Soma, Function groups in Kleinian groups, Math. Ann. 292 (1992), 181-190.

13. P. Susskind, Kleinian groups with intersecting limit sets, J. Analyse Math. 52 (1989), 26-38.

14. P. Susskind and G. A. Swarup, Limit sets of geometrically finite hyperbolic groups, Amer. J. Math. 114 (1992), 233-250.

Department of Mathematics, Rice University, Houston, Texas 77251

E-mail address: jwa@math.rice.edu 